\title{
DISTANCE BETWEEN METRIC MEASURE SPACES AND DISTANCE MATRIX DISTRIBUTIONS
}

\author{
By \\ Ryunosuke Ozawa
}

\begin{abstract}
We study the Prohorov distance between the distance matrix distributions of two metric measure spaces. We prove that it is not smaller than 1-box distance between two metric measure spaces and also prove that it is not larger than 0-box distance between two metric measure spaces.
\end{abstract}

\section{Introduction}

In this paper, we consider the relation between the box distance function and the distance matrix distribution. Here, a metric measure space $X:=\left(X, d_{X}, \mu_{X}\right)$ is a complete separable metric space $\left(X, d_{X}\right)$ equipped with a Borel probability measure $\mu_{X}$. The box distance function $\square_{\lambda}$, which was introduced by Gromov [5], is a natural distance function between two metric measure spaces for any $\lambda \geq 0$. Define the distance map $K_{r}^{X}$ from $X^{r}$ to $M_{r}(\mathbf{R})$, the set of all real square matrices of order $r$ with $l_{\infty}$-norm $\|\cdot\|_{\infty}$, by $K_{r}^{X}\left(x_{1}, \ldots, x_{r}\right):=\left(d_{X}\left(x_{k}, x_{l}\right)\right)_{k, l=1, \ldots, r}$. The $r$-dimensional distance matrix distribution $\mu_{r}^{X}$ is the push-forward measure of the $r$-times product measure of $\mu_{X}$ by $K_{r}^{X} \cdot \mu_{r}^{X}$ is a Borel probability measure on $M_{r}(\mathbf{R})$. We denote by $\mathscr{X}$ the set of isomorphism classes of metric measure spaces. Gromov [5] developed a theory of convergence of metric measure spaces in $\mathscr{X}$. He proved that the infinite-dimensional distance matrix distribution $\underline{\mu}_{\infty}^{X}$ is a complete invariant of metric measure spaces (see Theorem 2.3). On the other hand, Greven-Pfaffelhuber-Winter [4] developed a theory of convergence of metric measure spaces and the probabilistic theory on $\mathscr{X}$, independently of Gromov's

2010 Mathematics Subject Classification: Primary 53C23, 28 D05.

Key words and phrases: mm-space, box distance function, distance matrix distribution.

This work was partially supported by Research Fellowships of the Japan Society for the Promotion of Science for Young Scientists.

Received July 16, 2013.

Revised September 3, 2014. 
work. They introduced the Gromov-Prohorov metric $d_{\mathrm{GPr}}$ and proved that the topology of $d_{\mathrm{GPr}}$ is compatible with the weak convergence of infinite-dimensional distance matrix distributions (see Theorem 2.12). After that, Löhr [7] showed that $\square_{\lambda}(\lambda>0)$ and $d_{\mathrm{GPr}}$ are bi-Lipschitz equivalent to each other (see Theorem 2.16). Thus, the topology of the box distance function $\square_{\lambda}(\lambda>0)$ is compatible with the weak convergence of infinite-dimensional distance matrix distributions.

Our purpose is to estimate the box distance between two metric measure spaces $X$ and $Y$ by its the distance matrix distributions $\underline{\mu}_{\infty}^{X}$ and $\underline{\mu}_{\infty}^{Y}$. In this paper, we consider the distance between two distance matrix distributions $\underline{\mu}_{\infty}^{X}$ and $\underline{\mu}_{\infty}^{Y}$ of two metric measure spaces $X$ and $Y$. Moreover, we interpret it as a new metric on $\mathscr{X}$.

Definition 1.1. Let $X, Y \in \mathscr{X}$. We define the distance $d_{l_{\infty}-\operatorname{Pr}}(X, Y)$ to be the Prohorov distance between $\underline{\mu}_{\infty}^{X}$ and $\underline{\mu}_{\infty}^{Y}$ with respect to the $l_{\infty}$-norm on $M_{\infty}(\mathbf{R})$.

Remark 1.2. (1) Symmetry and triangle inequality of Prohorov metric (see Proposition 2.5) and Theorem 2.3 imply that $d_{l_{\infty}-\operatorname{Pr}}$ is a metric on $\mathscr{X}$.

(2) The $l_{\infty}$-norm $\|\cdot\|_{\infty}$ may take the value $\infty$ on $M_{\infty}(\mathbf{R})$ but the set of Borel probability measures on $M_{\infty}(\mathbf{R})$ equipped with the Prohorov metric with respect to the $l_{\infty}$-norm is a metric space.

(3) $d_{l_{\infty}-\operatorname{Pr}}(X, Y) \leq 1$ for any two mm-spaces $X$ and $Y$.

We study the relation between the box distance $\unrhd_{\lambda}(X, Y)$ and $d_{l_{\infty}-\operatorname{Pr}}(X, Y)$. Our main result is stated as follows.

THEOREM 1.3. Let $X, Y \in \mathscr{X}$. We have

$$
\unrhd_{1}(X, Y) \leq d_{l_{\infty}-\operatorname{Pr}}(X, Y) \leq \unrhd_{0}(X, Y) .
$$

We next compare the topologies induced from $\unrhd_{1}$ and $d_{l_{\infty}-\operatorname{Pr}}$.

Proposition 1.4. Let $X:=\left(\left\{p_{1}\right\}, \delta_{p_{1}}\right) \quad$ and $\quad X_{n}:=\left(\left\{p_{1}, p_{2}\right\}, d_{\left\{p_{1}, p_{2}\right\}}\right.$, $\left.\left(1-n^{-1}\right) \delta_{p_{1}}+n^{-1} \delta_{p_{2}}\right)$, where $d_{\left\{p_{1}, p_{2}\right\}}\left(p_{1}, p_{2}\right):=1$. We denote by $\delta_{p_{i}}$ the Dirac measure at $p_{i}(i=1,2)$. Then $\square_{1}\left(X_{n}, X\right)=n^{-1}$ but $d_{l_{\infty}-\operatorname{Pr}}\left(X_{n}, X\right)=\square_{0}\left(X_{n}, X\right)=1$ for any $n \geq 2$.

This proposition means that $\underline{\mu}_{\infty}^{X_{n}}$ converges to $\underline{\mu}_{\infty}^{X}$ weakly as $n \rightarrow \infty$ but the Prohorov distance between $\underline{\mu}_{\infty}^{X_{n}}$ and $\underline{\mu}_{\infty}^{X}$ is one for any $n \geq 2$ and implies the next corollary. 
COROLlary 1.5. $\left(\mathscr{X}, d_{l_{\infty}-\operatorname{Pr}}\right)$ and $\left(\mathscr{X}, \square_{1}\right)$ are not homeomorphic to each other by the identity map.

Corollary 1.5 seems not to fit to the fact that the topology of the Prohorov metric is compatible with the weak convergence of probability measures on the complete separable metric space (see Theorem 2.7). Since $\left(M_{\infty}(\mathbf{R}),\|\cdot\|_{\infty}\right)$ is not separable, Corollary 1.5 does not contradictory. We do not know if the topology generated by $\square_{0}$ coincides with the topology induced from $d_{l_{\infty} \text {-Pr }}$ or not.

\section{Preliminaries}

2.1. Metric Measure Space. Let $\left(X, \mathcal{O}_{X}\right)$ be a topological space. We denote by $\mathscr{B}(X)$ the Borel $\sigma$-algebra and $\mathscr{M}(X)$ the set of all Borel probability measures on $X$. Let $\mu$ be a Borel probability measure on $X$. Recall that the support of $\mu$, $\operatorname{supp}(\mu)$, is the smallest closed set $\operatorname{supp}(\mu) \subset X$ such that $\mu(X \backslash \operatorname{supp}(\mu))=0$. The push forward of $\mu$ by a measurable map $\varphi$ from $X$ into another topological space $\left(Y, \mathcal{O}_{Y}\right)$ is the Borel probability measure $\varphi_{*} \mu \in \mathscr{M}(Y)$ defined by

$$
\varphi_{*} \mu(A):=\mu\left(\varphi^{-1}(A)\right),
$$

for all $A \in \mathscr{B}(Y)$.

Definition 2.1 (Metric measure space). A triple $X:=\left(X, d_{X}, \mu_{X}\right)$ is called a metric measure space (or mm-space) if $\left(X, d_{X}\right)$ is a complete separable metric space and if $\mu_{X}$ is a Borel probability measure on $X$. Two mm-spaces $\left(X, d_{X}, \mu_{X}\right)$ and $\left(Y, d_{Y}, \mu_{Y}\right)$ are isomorphic if there exists an isometry $\varphi$ between the supports of $\mu_{X}$ on $\left(X, d_{X}\right)$ and of $\mu_{Y}$ on $\left(Y, d_{Y}\right)$ such that $\mu_{Y}=\varphi_{*} \mu_{X}$. We write $\mathscr{X}$ by the set of isomorphism classes of mm-spaces.

Let $M_{\infty}(\mathbf{R})$ be the set of all real square matrices of infinite order equipped with the coarsest topology such that the natural projection $\operatorname{pr}_{\infty, r}: M_{\infty}(\mathbf{R}) \rightarrow$ $M_{r}(\mathbf{R})$ is continuous for any $r \in \mathbf{R}$.

Definition 2.2 (Distance matrix distribution). Let $X=\left(X, d_{X}, \mu_{X}\right) \in \mathscr{X}$ and $r \in \mathbf{N} \cup\{\infty\}$. Define a map $K_{r}^{X}: X^{r} \rightarrow M_{r}(\mathbf{R})$ by

$$
K_{r}^{X}\left(x_{1}, \ldots, x_{r}\right):=\left(d_{X}\left(x_{k}, x_{l}\right)\right)_{k, l=1, \ldots, r},
$$


and the r-dimensional distance matrix distribution $\underline{\mu}_{r}^{X}$ of $X$ by

$$
\underline{\mu}_{r}^{X}:=\left(K_{r}^{X}\right)_{*} \mu_{X}^{\otimes r},
$$

where $\mu_{X}^{\otimes r}$ is the $r$ times product measure of $\mu_{X}$.

THEOREM 2.3 (mm-Reconstruction theorem, [5, Section $3 \frac{1}{2} .5$, Section $\left.3 \frac{1}{2} .7\right]$, [8, Section 2, Theorem], [6, Theorem 2.1]). Let $X, Y \in \mathscr{X}$. The following (1), (2), and (3) are equivalent to each other.

(1) $X$ and $Y$ are isomorphic to each other.

(2) $\underline{\mu}_{r}^{X}=\underline{\mu}_{r}^{Y}$ for all $r \in \mathbf{N}$.

(3) $\underline{\mu}_{\infty}^{X}=\underline{\mu}_{\infty}^{Y}$.

This theorem means that the infinite-dimensional distance matrix distribution is a complete invariant of mm-spaces.

2.2. Gromov-Prohorov Metric. Let $\left(X, d_{X}\right)$ be a metric space. For a real number $r>0$ and a subset $A \subset X$, we set $B_{r}(A):=\left\{x \in X \mid d_{X}(x, A)<r\right\}$, where $d_{X}(x, A):=\inf _{x^{\prime} \in A} d_{X}\left(x, x^{\prime}\right)$.

Definition 2.4 (Prohorov metric). Define the Prohorov metric $d_{\mathrm{Pr}}^{\left(X, d_{X}\right)}$ on $\mathscr{M}(X)$ by

$$
d_{\mathrm{Pr}}^{\left(X, d_{X}\right)}(\mu, v):=\inf \left\{\varepsilon>0 \mid \mu(A) \leq v\left(B_{\varepsilon}(A)\right)+\varepsilon, \text { for all } A \in \mathscr{B}(X)\right\}
$$

for $\mu, v \in \mathscr{M}(X)$.

Note that $d_{\operatorname{Pr}}^{\left(X, d_{X}\right)}(\mu, v) \leq 1$ for any two Borel probability measures $\mu$ and $v$ on $X$.

Proposition 2.5 ([2, Lemma 3.1.1]). $\quad\left(\mathscr{M}(X), d_{\mathrm{Pr}}^{\left(X, d_{X}\right)}\right)$ is a metric space.

Definition 2.6 (Weak convergence). We say that a sequence $\left\{\mu_{n}\right\}_{n=1}^{\infty}$ of Borel probability measures on $X$ converges weakly to a Borel probability measure $\mu$ on $X$ and write $\mu_{n} \rightarrow \mu$ weakly as $n \rightarrow \infty$ if

$$
\lim _{n \rightarrow \infty} \int_{X} f(x) d \mu_{n}=\int_{X} f(x) d \mu
$$

for any bounded continuous function $f: X \rightarrow \mathbf{R}$. 
THEOREM 2.7 ([2, Theorem 3.3.1]). Let $\left(X, d_{X}\right)$ be a separable metric space, $\left\{\mu_{n}\right\}_{n=1}^{\infty}$ a sequence of Borel probability measures on $X$, and $\mu$ a Borel probability measure on $X$. Then

$$
\lim _{n \rightarrow \infty} d_{\operatorname{Pr}}^{\left(X, d_{X}\right)}\left(\mu_{n}, \mu\right)=0
$$

if and only if

$$
\mu_{n} \rightarrow \mu \text { weakly as } n \rightarrow \infty \text {. }
$$

For $\mu, v \in \mathscr{M}(X)$, we say $\mu \leq v$ if $\mu(A) \leq v(A)$ for any $A \in \mathscr{B}(X)$. A finite Borel measure $\pi$ on $X \times X$ is called a partial transport plan from $\mu \in \mathscr{M}(X)$ to $v \in \mathscr{M}(X)$ if $\left(\mathrm{pr}_{1}\right)_{*} \pi \leq \mu$ and $\left(\mathrm{pr}_{2}\right)_{*} \pi \leq v$, where $\mathrm{pr}_{i}: X \times X \rightarrow X, i=1,2$, are the projections defined by $\operatorname{pr}_{1}\left(x, x^{\prime}\right)=x, \operatorname{pr}_{2}\left(x, x^{\prime}\right)=x^{\prime}$. For a partial transport plan $\pi$ from $\mu$ to $v$, we define the deficiency def $\pi$ of $\pi$ by def $\pi:=1-\pi(X \times X)$. For $\varepsilon \geq 0$, the partial transport plan $\pi$ is called an $\varepsilon$-transport plan from $\mu$ to $v$ if

$$
\operatorname{supp}(\pi) \subset X(\varepsilon):=\left\{\left(x, x^{\prime}\right) \in X \times X \mid d_{X}\left(x, x^{\prime}\right) \leq \varepsilon\right\}
$$

Definition 2.8 (Transportation distance). Let $\mu, v \in \mathscr{M}(X)$. Define the Transportation distance $\operatorname{Tra}^{\left(X, d_{X}\right)}$ between $\mu$ and $v$ by

$$
\begin{gathered}
\operatorname{Tra}^{\left(X, d_{X}\right)}(\mu, v):=\inf \{\varepsilon>0 \mid \text { there exists an } \varepsilon \text {-transport plan } \pi \\
\text { from } \mu \text { to } v \text { satisfying def } \pi \leq \varepsilon\} .
\end{gathered}
$$

THEOREM 2.9 (Strassen's theorem, [9, Corollary 1.28]). Let $\left(X, d_{X}\right)$ be a complete separable metric space. For any $\mu, v \in \mathscr{M}(X)$, we have

$$
d_{\mathrm{Pr}}^{\left(X, d_{X}\right)}(\mu, v)=\operatorname{Tra}^{\left(X, d_{X}\right)}(\mu, v)
$$

Next, we define the Gromov-Prohorov metric on $\mathscr{X}$.

Definition 2.10 (Gromov-Prohorov metric, [4]). Let $X=\left(X, d_{X}, \mu_{X}\right), Y=$ $\left(Y, d_{Y}, \mu_{Y}\right)$ be two mm-spaces. Define the Gromov-Prohorov metric $d_{\mathrm{GPr}}$ on $\mathscr{X}$ by

$$
d_{\mathrm{GPr}}(X, Y):=\inf _{\left(\varphi_{X}, \varphi_{Y}, Z\right)} d_{\mathrm{Pr}}^{\left(Z, d_{Z}\right)}\left(\left(\varphi_{X}\right)_{*} \mu_{X},\left(\varphi_{Y}\right)_{*} \mu_{Y}\right),
$$

where the infimum is taken over all isometric embeddings $\varphi_{X}$ and $\varphi_{Y}$ from $\operatorname{supp}\left(\mu_{X}\right)$ and $\operatorname{supp}\left(\mu_{Y}\right)$, respectively, into some common metric space $\left(Z, d_{Z}\right)$. 
Note that $d_{\mathrm{GPr}}(X, Y) \leq 1$ for any two mm-spaces $X$ and $Y$.

THEOREM 2.11 ([4, Theorem 1]). ( $\left.\mathscr{X}, d_{\mathrm{GPr}}\right)$ is a metric space.

Theorem 2.12 ([4, Theorem 5]). Let $X, X_{1}, X_{2}, \ldots \in \mathscr{X}$. Then

$$
\lim _{n \rightarrow \infty} d_{\mathrm{GPr}}\left(X_{n}, X\right)=0
$$

if and only if

$$
\underline{\mu}_{\infty}^{X_{n}} \rightarrow \underline{\mu}_{\infty}^{X} \text { weakly as } n \rightarrow \infty .
$$

2.3. Gromov's Box Distance. We denote by $\mathscr{L}$ the Lebesgue measure on $[0,1]$. For any mm-space $X$ there exists a Borel measurable map $p_{X}:[0,1] \rightarrow X$ with $\left(p_{X}\right)_{*} \mathscr{L}=\mu_{X}$ (see [1, Theorem 9.4.7]). We call such a map $p_{X}$ a parameter of $X$. Note that a parameter of $X$ is not unique in general. For a parameter $p_{X}$ of $X$, we define a function $\left(p_{X}\right)^{*} d_{X}:[0,1] \times[0,1] \rightarrow \mathbf{R}$ by $\left(p_{X}\right)^{*} d_{X}\left(s, s^{\prime}\right):=$ $d_{X}\left(p_{X}(s), p_{X}\left(s^{\prime}\right)\right)$.

Definition 2.13 (Box distance, [5]). Let $\lambda \geq 0$, and $X=\left(X, d_{X}, \mu_{X}\right), \quad Y=$ $\left(Y, d_{Y}, \mu_{Y}\right) \in \mathscr{X}$. Define the box distance between $X$ and $Y$ by

$$
\begin{array}{r}
\square_{\lambda}(X, Y):=\inf _{\left(p_{X}, p_{Y}\right)}\left\{\varepsilon>0 \mid \text { there exists } T_{\varepsilon} \in \mathscr{B}([0,1]) \text { such that } \mathscr{L}\left(T_{\varepsilon}\right) \geq 1-\lambda \varepsilon\right. \\
\text { and } \left.\left|\left(p_{X}\right)^{*} d_{X}\left(s, s^{\prime}\right)-\left(p_{Y}\right)^{*} d_{Y}\left(s, s^{\prime}\right)\right| \leq \varepsilon \text { for all } s, s^{\prime} \in T_{\varepsilon}\right\},
\end{array}
$$

where the infimum is taken over all parameters $p_{X}:[0,1] \rightarrow X$ and $p_{Y}:[0,1]$ $\rightarrow Y$.

Note that $\square_{\lambda}(X, Y) \leq 1 / \lambda$ for any two mm-spaces $X, Y$, and $\lambda>0$.

Theorem 2.14 ([5, Section $\left.3 \frac{1}{2} \cdot 6\right],\left[3\right.$, Theorem 3.1.8]). $\left(\mathscr{X}, \square_{\lambda}\right)$ is a metric space for any $\lambda \geq 0$.

It is easy to see that $\square_{\lambda}$ for all $\lambda>0$ are bi-Lipschitz equivalent to each other and $\unrhd_{1} \leq \unrhd_{0}$.

Proposition 2.15 ([5, Section $\left.\left.3 \frac{1}{2} \cdot 10\right]\right)$. Let $\left(X, d_{X}\right)$ be a complete separable metric space and $\mu, v \in \mathscr{M}(X)$. Then we have

$$
\unrhd_{1}\left(\left(X, d_{X}, \mu\right),\left(X, d_{X}, v\right)\right) \leq d_{\mathrm{Pr}}^{\left(X, d_{X}\right)}(\mu, v) .
$$


Finally, we consider the relation between the Gromov-Prohorov metric and the box distance function.

Theorem 2.16 ([7, Corollary 6]). For any $X, Y \in \mathscr{X}$, we have

$$
d_{\mathrm{GPr}}(X, Y) \leq \unrhd_{1}(X, Y) \leq 2 d_{\mathrm{GPr}}(X, Y) .
$$

Moreover, $\left(\mathscr{X}, d_{\mathrm{GPr}}\right)$ and $\left(\mathscr{X}, \square_{1}\right)$ are homeomorphic to each other.

REMARK 2.17. (1) Löhr [7] proved that $d_{\mathrm{GPr}}=\frac{1}{2} \unrhd_{1 / 2}$ on $\mathscr{X}$. This implies the inequality (2.1).

(2) Theorem 2.12 and Theorem 2.16 imply that $X_{n} \square_{1}$-converges $X$ as $n \rightarrow \infty$ if and only if $\underline{\mu}_{\infty}^{X_{n}}$ converses weakly to $\underline{\mu}_{\infty}^{X}$ as $n \rightarrow \infty$. The proof of this statement is omitted in the original article (see [5, Section $\left.3 \frac{1}{2} .14\right]$ ).

\section{Box Distance and Distance Matrix Distribution}

In this section, we give the proof of Theorem 1.3 .

Let $r, r^{\prime} \in \mathbf{N} \cup\{\infty\}$ with $r \geq r^{\prime}$. Define the projection $\operatorname{pr}_{r, r^{\prime}}: M_{r}(\mathbf{R}) \rightarrow M_{r^{\prime}}(\mathbf{R})$ by

$$
\operatorname{pr}_{r, r^{\prime}}\left(\left(a_{k l}\right)_{k, l=1, \ldots, r}\right):=\left(a_{k l}\right)_{k, l=1, \ldots, r^{\prime}}
$$

Lemma 3.1 ([6, Lemma 2.2]). Let $X \in \mathscr{X}$ and $r \in \mathbf{N}$. We have $\left(\operatorname{pr}_{r+1, r}\right)_{*} \underline{\mu}_{r+1}^{X}=$ $\underline{\mu}_{r}^{X}$ and $\left(\mathrm{pr}_{\infty, r}\right)_{*} \underline{\mu}_{\infty}^{X}=\underline{\mu}_{r}^{X}$.

Lemma 3.2. Let $X, Y \in \mathscr{X}$. Then $d_{\mathrm{Pr}}^{\left(M_{r}(\mathbf{R}),\|\cdot\|_{\infty}\right)}\left(\underline{\mu}_{r}^{X}, \underline{\mu}_{r}^{Y}\right)$ is monotone nondecreasing in $r \in \mathbf{N}$. In particular,

$$
\sup _{r \in \mathbf{N}} d_{\operatorname{Pr}}^{\left(M_{r}(\mathbf{R}),\|\cdot\|_{\infty}\right)}\left(\underline{\mu}_{r}^{X}, \underline{\mu}_{r}^{Y}\right)=\lim _{r \rightarrow \infty} d_{\operatorname{Pr}}^{\left(M_{r}(\mathbf{R}),\|\cdot\|_{\infty}\right)}\left(\underline{\mu}_{r}^{X}, \underline{\mu}_{r}^{Y}\right) .
$$

ProOF. Let $\varepsilon>0$ satisfy $d_{\mathrm{Pr}}^{\left(M_{r+1}(\mathbf{R}),\|\cdot\|_{\infty}\right)}\left(\underline{\mu}_{r+1}^{X}, \underline{\mu}_{r+1}^{Y}\right)<\varepsilon$. By the definition of Prohorov metric, we get $\underline{\mu}_{r+1}^{X}(A) \leq \underline{\mu}_{r+1}^{Y}\left(B_{\varepsilon}(A)\right)+\varepsilon$ for all $A \in \mathscr{B}\left(M_{r+1}(\mathbf{R})\right)$. Since $\operatorname{pr}_{r+1, r}^{-1}\left(A^{\prime}\right) \in \mathscr{B}\left(M_{r+1}(\mathbf{R})\right)$ for any $A^{\prime} \in \mathscr{B}\left(M_{r}(\mathbf{R})\right)$, we have

$$
\underline{\mu_{r+1}^{X}}\left(\operatorname{pr}_{r+1, r}^{-1}\left(A^{\prime}\right)\right) \leq \underline{\mu}_{r+1}^{Y}\left(B_{\varepsilon}\left(\operatorname{pr}_{r+1, r}^{-1}\left(A^{\prime}\right)\right)\right)+\varepsilon .
$$

Obviously, $B_{\varepsilon}\left(\operatorname{pr}_{r+1, r}^{-1}\left(A^{\prime}\right)\right)=\operatorname{pr}_{r+1, r}^{-1}\left(B_{\varepsilon}\left(A^{\prime}\right)\right)$. Therefore,

$$
\underline{\mu}_{r+1}^{X}\left(\operatorname{pr}_{r+1, r}^{-1}\left(A^{\prime}\right)\right) \leq \underline{\mu}_{r+1}^{Y}\left(\operatorname{pr}_{r+1, r}^{-1}\left(B_{\varepsilon}\left(A^{\prime}\right)\right)\right)+\varepsilon .
$$


By Lemma 3.1, this means

$$
\underline{\mu}_{r}^{X}\left(A^{\prime}\right) \leq \underline{\mu}_{r}^{Y}\left(B_{\varepsilon}\left(A^{\prime}\right)\right)+\varepsilon,
$$

then we obtain $d_{\operatorname{Pr}}^{\left(M_{r}(\mathbf{R}),\|\cdot\|_{\infty}\right)}\left(\underline{\mu}_{r}^{X}, \underline{\mu}_{r}^{Y}\right) \leq \varepsilon$. By the arbitrariness of $\varepsilon$, we have $d_{\mathrm{Pr}}^{\left(M_{r}(\mathbf{R}),\|\cdot\|_{\infty}\right)}\left(\underline{\mu}_{r}^{X}, \underline{\mu}_{r}^{Y}\right) \leq d_{\mathrm{Pr}}^{\left(M_{r+1}(\mathbf{R}),\|\cdot\|_{\infty}\right)}\left(\underline{\mu}_{r+1}^{X}, \underline{\mu}_{r+1}^{Y}\right)$.

Lemma 3.3. Let $X, Y \in \mathscr{X}$. Then we have

$$
d_{l_{\infty}-\operatorname{Pr}}(X, Y)=\lim _{r \rightarrow \infty} d_{\operatorname{Pr}}^{\left(M_{r}(\mathbf{R}),\|\cdot\|_{\infty}\right)}\left(\underline{\mu}_{r}^{X}, \underline{\mu}_{r}^{Y}\right) .
$$

Proof. The inequality

$$
\lim _{r \rightarrow \infty} d_{\operatorname{Pr}}^{\left(M_{r}(\mathbf{R}),\|\cdot\|_{\infty}\right)}\left(\underline{\mu}_{r}^{X}, \underline{\mu}_{r}^{Y}\right) \leq d_{l_{\infty}-\operatorname{Pr}}(X, Y)
$$

is obtained in the same way as in the proof of Lemma 3.2.

Next, we prove the inequality

$$
d_{l_{\infty}-\operatorname{Pr}}(X, Y) \leq \sup _{r \in \mathbf{N}} d_{\operatorname{Pr}}^{\left(M_{r}(\mathbf{R}),\|\cdot\|_{\infty}\right)}\left(\underline{\mu}_{r}^{X}, \underline{\mu}_{r}^{Y}\right)
$$

by Lemma 3.2. Let $\varepsilon^{\prime}>0$ satisfy $d_{\mathrm{Pr}}^{\left(M_{r}(\mathbf{R}),\|\cdot\|_{\infty}\right)}\left(\underline{\mu}_{r}^{X}, \underline{\mu}_{r}^{Y}\right)<\varepsilon^{\prime}$ for any $r \in \mathbf{N}$, and $A^{\prime} \in \mathscr{B}\left(M_{\infty}(\mathbf{R})\right)$. By the definition of Prohorov metric, Lemma 3.1 and $\operatorname{pr}_{\infty, r}^{-1}\left(B_{\varepsilon^{\prime}}(A)\right)=B_{\varepsilon^{\prime}}\left(\operatorname{pr}_{\infty, r}^{-1}(A)\right)$ for any $A \in \mathscr{B}\left(M_{r}(\mathbf{R})\right)$, we get

$$
\underline{\mu}_{\infty}^{X}\left(\operatorname{pr}_{\infty, r}^{-1}\left(\operatorname{pr}_{\infty, r}\left(A^{\prime}\right)\right)\right) \leq \underline{\mu}_{\infty}^{Y}\left(B_{\varepsilon^{\prime}}\left(\operatorname{pr}_{\infty, r}^{-1}\left(\operatorname{pr}_{\infty, r}\left(A^{\prime}\right)\right)\right)\right)+\varepsilon^{\prime} .
$$

Using the continuity of measure for $\left\{\bigcap_{n=1}^{r} \operatorname{pr}_{\infty, n}^{-1}\left(\operatorname{pr}_{\infty, n}\left(A^{\prime}\right)\right)\right\}_{r=1}^{\infty}$, we have

$$
\lim _{r \rightarrow \infty} \underline{\mu}_{\infty}^{X}\left(\operatorname{pr}_{\infty, r}^{-1}\left(\operatorname{pr}_{\infty, r}\left(A^{\prime}\right)\right)\right)=\underline{\mu}_{\infty}^{X}\left(A^{\prime}\right)
$$

and

$$
\lim _{r \rightarrow \infty} \underline{\mu}_{\infty}^{Y}\left(B_{\varepsilon^{\prime}}\left(\operatorname{pr}_{\infty, r}^{-1}\left(\operatorname{pr}_{\infty, r}\left(A^{\prime}\right)\right)\right)\right)=\underline{\mu}_{\infty}^{Y}\left(B_{\varepsilon^{\prime}}\left(A^{\prime}\right)\right) .
$$

(3.3) and (3.4) lead to $\underline{\mu}_{\infty}^{X}\left(A^{\prime}\right) \leq \underline{\mu}_{\infty}^{Y}\left(B_{\varepsilon^{\prime}}\left(A^{\prime}\right)\right)+\varepsilon^{\prime}$. Then this means

$$
d_{l_{\infty}-\operatorname{Pr}}(X, Y) \leq \varepsilon^{\prime} .
$$

We obtain (3.2).

Combining two inequalities (3.1) and (3.2), we have the lemma.

To prove Theorem 1.3, we need a uniformly distributed sequence. 
Definition 3.4 (Uniformly distributed sequence). Let $X \in \mathscr{X}$ and $\left\{x_{i}\right\}_{i=1}^{\infty} \subset X$. $\left\{x_{i}\right\}_{i=1}^{\infty}$ is called a uniformly distributed sequence of $X$ if

$$
\frac{1}{n} \sum_{i=1}^{n} \delta_{x_{i}} \rightarrow \mu_{X} \text { weakly as } n \rightarrow \infty,
$$

where $\delta_{x_{i}}$ is the Dirac measure at $x_{i}$. We write $E_{X}$ by the set of all uniformly distributed sequences of $X$.

Consider $E_{X}$ as a subset of $X^{\mathbf{N}}$. The next lemma means that there are many uniformly distributed sequences of $X$.

Lemma 3.5 ([6, Lemma 2.4]). Let $X \in \mathscr{X}$. We have $\mu_{X}^{\otimes \mathbf{N}}\left(E_{X}\right)=1$ and in particular, $\underline{\mu}_{\infty}^{X}\left(K_{\infty}^{X}\left(E_{X}\right)\right)=1$.

Finally, we prove the main theorem.

Proof OF TheOrem 1.3. We first prove the inequality $\square_{1}(X, Y) \leq$ $d_{l_{\infty}-\operatorname{Pr}}(X, Y)$. This is trivial in the case of $d_{l_{\infty}-\operatorname{Pr}}(X, Y)=1$. Let $0<\varepsilon<1$ satisfy $d_{l_{\infty}-\operatorname{Pr}}(X, Y)<\varepsilon$. We apply the definition of $d_{l_{\infty}-\operatorname{Pr}}$ for $A=K_{\infty}^{X}\left(E_{X}\right)$ and use Lemma 3.5 to have

$$
\underline{\mu}_{\infty}^{Y}\left(B_{\varepsilon}\left(K_{\infty}^{X}\left(E_{X}\right)\right)\right) \geq 1-\varepsilon>0 .
$$

By $\underline{\mu}_{\infty}^{Y}\left(K_{\infty}^{Y}\left(E_{Y}\right)\right)=1$, we have $B_{\varepsilon}\left(K_{\infty}^{X}\left(E_{X}\right)\right) \cap K_{\infty}^{X}\left(E_{Y}\right) \neq \varnothing$. Then there exist two sequences $\left\{x_{i}\right\}_{i=1}^{\infty} \in E_{X}$ and $\left\{y_{i}\right\}_{i=1}^{\infty} \in E_{Y}$ such that

$$
\left|d_{X}\left(x_{i}, x_{j}\right)-d_{Y}\left(y_{i}, y_{j}\right)\right|<\varepsilon
$$

for all $i, j \in \mathbf{N}$. Define mm-spaces $X_{n}$ and $Y_{n}$ by

$$
X_{n}:=\left(X, d_{X}, \frac{1}{n} \sum_{i=1}^{n} \delta_{x_{i}}\right), \quad Y_{n}:=\left(Y, d_{Y}, \frac{1}{n} \sum_{i=1}^{n} \delta_{y_{i}}\right) .
$$

From the definition of uniformly distributed sequence and Proposition 2.15, for any $\delta>0$, there exists a number $n_{0} \in \mathbf{N}$ such that $\square_{1}\left(X_{n}, X\right)<\delta$ and $\square_{1}\left(Y_{n}, Y\right)<\delta$ for any $n \geq n_{0}$. Define two parameters $p_{X_{n}}$ and $p_{Y_{n}}$ of $X_{n}$ and $Y_{n}$ by

$$
\begin{aligned}
& p_{X_{n}}(s):= \begin{cases}x_{i} & \text { if } s \in\left[\frac{i-1}{n}, \frac{i}{n}\right), i=1, \ldots, n, \\
x_{n} & \text { if } s=1,\end{cases} \\
& p_{Y_{n}}(s):= \begin{cases}y_{i} & \text { if } s \in\left[\frac{i-1}{n}, \frac{i}{n}\right), i=1, \ldots, n, \\
y_{n} & \text { if } s=1 .\end{cases}
\end{aligned}
$$


We then have

$$
\left|\left(p_{X_{n}}\right)^{*} d_{X}\left(s, s^{\prime}\right)-\left(p_{Y_{n}}\right)^{*} d_{Y}\left(s, s^{\prime}\right)\right| \leq \varepsilon
$$

for any $s, s^{\prime} \in[0,1]$. This implies $\square_{1}\left(X_{n}, Y_{n}\right)<\varepsilon$. Thus, we have

$$
\begin{aligned}
\unrhd_{1}(X, Y) & \leq \unrhd_{1}\left(X, X_{n}\right)+\unrhd_{1}\left(X_{n}, Y_{n}\right)+\unrhd_{1}\left(Y_{n}, Y\right) \\
& <2 \delta+\varepsilon .
\end{aligned}
$$

By the arbitrariness of $\delta$ and $\varepsilon$, we have $\emptyset_{1}(X, Y) \leq d_{l_{\infty}-\operatorname{Pr}}(X, Y)$.

We next prove the inequality $d_{l_{\infty}-\operatorname{Pr}}(X, Y) \leq \square_{0}(X, Y)$. Let $\varepsilon>0$ satisfy $\square_{0}(X, Y)<\varepsilon$. From the definition of box distance, there exist two parameters $p_{X}, p_{Y}$ of $X, Y$ and a Borel set $T_{\varepsilon}$ on $[0,1]$ such that $\mathscr{L}\left(T_{\varepsilon}\right)=1$ and

$$
\left|\left(p_{X}\right)^{*} d_{X}\left(s, s^{\prime}\right)-\left(p_{Y}\right)^{*} d_{Y}\left(s, s^{\prime}\right)\right|<\varepsilon
$$

for all $s, s^{\prime} \in T_{\varepsilon}$. Define the map $\xi_{r}:\left(T_{\varepsilon}\right)^{r} \rightarrow M_{r}(\mathbf{R}) \times M_{r}(\mathbf{R})$ by

$$
\xi_{r}\left(s_{1}, \ldots, s_{r}\right):=\left(K_{r}^{X}\left(p_{X}\left(s_{1}\right), \ldots, p_{X}\left(s_{r}\right)\right), K_{r}^{Y}\left(p_{Y}\left(s_{1}\right), \ldots, p_{Y}\left(s_{r}\right)\right)\right) .
$$

We set $\pi_{r}:=\left(\xi_{r}\right)_{*} \mathscr{L}^{\otimes r}$. This belongs to $\mathscr{M}\left(M_{r}(\mathbf{R}) \times M_{r}(\mathbf{R})\right)$. We will prove that $\pi_{r}$ is an $\varepsilon$-transportation from $\underline{\mu}_{r}^{X}$ to $\underline{\mu}_{r}^{Y}$ and def $\pi_{r} \leq \varepsilon$. Obviously,

$$
\operatorname{supp}\left(\pi_{r}\right) \subset \xi_{r}\left(\left(T_{\varepsilon}\right)^{r}\right) \subset M_{r}(\mathbf{R})(\varepsilon) .
$$

Define two projections $\operatorname{pr}_{1}: M_{r}(\mathbf{R}) \times M_{r}(\mathbf{R}) \rightarrow M_{r}(\mathbf{R}), \operatorname{pr}_{2}: M_{r}(\mathbf{R}) \times M_{r}(\mathbf{R}) \rightarrow$ $M_{r}(\mathbf{R})$ by $\operatorname{pr}_{1}\left(\left(a_{i j}\right),\left(b_{i j}\right)\right):=\left(a_{i j}\right), \quad \operatorname{pr}_{2}\left(\left(a_{i j}\right),\left(b_{i j}\right)\right):=\left(b_{i j}\right)$. Then for all $A \in$ $\mathscr{B}\left(M_{r}(\mathbf{R})\right)$,

$$
\begin{aligned}
\left(\operatorname{pr}_{1}\right)_{*} \pi_{r}(A) & =\left(\operatorname{pr}_{1} \circ \xi_{r}\right)_{*} \mathscr{L}^{\otimes r}(A) \\
& =\mathscr{L}^{\otimes r}\left(\xi_{r}^{-1}\left(\operatorname{pr}_{1}^{-1}(A)\right)\right) \\
& =\mathscr{L}^{\otimes r}\left(\xi_{r}^{-1}\left(A \times M_{r}(\mathbf{R})\right)\right) \\
& =\mathscr{L}^{\otimes r}\left(\left\{\left(s_{1}, \ldots, s_{r}\right) \in\left(T_{\varepsilon}\right)^{r} \mid K_{r}^{X}\left(p_{X}\left(s_{1}\right), \ldots, p_{X}\left(s_{r}\right)\right) \in A\right\}\right) \\
& =\mu_{X}^{\otimes r}\left(\left\{\left(x_{1}, \ldots, x_{r}\right) \in X^{r} \mid K_{r}^{X}\left(x_{1}, \ldots, x_{r}\right) \in A\right\}\right) \\
& =\underline{\mu}_{r}^{X}(A) .
\end{aligned}
$$

This leads to $\left(\mathrm{pr}_{1}\right)_{*} \pi_{r}=\underline{\mu}_{r}^{X}$. In the same way, we get $\left(\mathrm{pr}_{2}\right)_{*} \pi_{r}=\underline{\mu}_{r}^{Y}$. Then $\pi_{r}$ is an $\varepsilon$-transportation from $\underline{\mu}_{r}^{X}$ to $\underline{\mu}_{r}^{Y}$ and $\operatorname{def} \pi_{r}=0 \leq \varepsilon$. This means that $\operatorname{Tra}^{\left(M_{r}(\mathbf{R}),\|\cdot\|_{\infty}\right)}\left(\underline{\mu}_{r}^{X}, \underline{\mu}_{r}^{Y}\right) \leq \varepsilon$. We get $\overline{d_{l_{\infty}-\operatorname{Pr}}}(X, Y) \leq \square_{0}(X, Y)$ by Theorem 2.9, the arbitrariness of $\varepsilon$, and Lemma 3.3.

Combining these two inequalities, we obtain the theorem. 
Proof of Proposition 1.4. First, we prove $\square_{1}\left(X_{n}, X\right)=n^{-1}$. For any parameter $p_{X_{n}}$ of $X_{n}, \mathscr{L}\left(p_{X_{n}}^{-1}\left(\left\{p_{1}\right\}\right)\right)=1-n^{-1}$. Then we have $d_{\left\{p_{1}, p_{2}\right\}}\left(p_{X_{n}}(s), p_{X_{n}}\left(s^{\prime}\right)\right)$ $=0$ for any $s, s^{\prime} \in p_{X_{n}}^{-1}\left(\left\{p_{1}\right\}\right)$. This means that $\square_{1}\left(X_{n}, X\right)=n^{-1}$.

It is obvious that $\square_{0}\left(X_{n}, X\right)=1$ for any $n \geq 2$.

Next, we prove $d_{l_{\infty}-\mathrm{Pr}}\left(X_{n}, X\right)=1$ for any $n \geq 2$. We set the Borel set $A_{r}$ on $M_{r}(\mathbf{R})$ by

$$
A_{r}:=\left\{\left(a_{i j}\right) \in M_{r}(\mathbf{R}) \mid \text { there exist } k, l \in\{1, \ldots, r\} \text { such that } a_{k l}=1\right\} .
$$

Then we have

$$
\begin{aligned}
\underline{\mu}_{r}^{X_{n}}\left(A_{r}\right) & =\mu_{X_{n}}^{\otimes r}\left(\left\{\left(x_{1}, \ldots, x_{r}\right) \in X_{n}^{r} \mid\left(x_{1}, \ldots, x_{r}\right) \neq\left(p_{i}, \ldots, p_{i}\right), i=1,2\right\}\right. \\
& =\sum_{k=1}^{r-1}\left(\begin{array}{c}
r \\
k
\end{array}\right) n^{-k}\left(1-n^{-1}\right)^{r-k} \\
& =1-n^{-r}-\left(1-n^{-1}\right)^{r} .
\end{aligned}
$$

It is obvious that $\underline{\mu}_{r}^{X}\left(A_{r}\right)=0$ and $\underline{\mu}_{r}^{X}\left(B_{1-n^{-r}-\left(1-n^{-1}\right)^{r}}\left(A_{r}\right)\right)=0$ for any $n \in \mathbf{N}$. Let $\varepsilon>0$. We have $\mu_{r}^{\overline{X_{n}}}\left(A_{r}\right) \leq \mu_{r}^{X}\left(B_{\varepsilon}\left(\overline{A_{r}}\right)\right)+\varepsilon$ if and only if $\varepsilon \geq 1-n^{-r}-\left(1-n^{-1}\right)^{r}$. This means that $d_{\mathrm{Pr}}^{\left(M_{r}(\mathbf{R}),\|\cdot\|_{\infty}\right)}\left(\mu_{r}^{X}, \mu_{r}^{X_{n}}\right) \geq 1-n^{-r}-\left(1-n^{-1}\right)^{r}$. For any $n \geq 2$, we get $d_{l_{\infty}-\operatorname{Pr}}\left(X_{n}, X\right)=1$ by Lemma 3.3 .

\section{Acknowledgements}

The author would like to thank Professor Takashi Shioya, Dr. Kazuki Ito, and Dr. Yu Kitabeppu for valuable discussions and many fruitful suggestions. $\mathrm{He}$ also thanks Professors Kei Funano and Takumi Yokota for their encouragement.

\section{References}

[1] V. I. Bogachev, Measure theory. Vol. I, II, Springer-Verlag, Berlin, 2007. MR2267655 (2008g:28002)

[2] Stewart N. Ethier and Thomas G. Kurtz, Markov processes, Wiley Series in Probability and Mathematical Statistics: Probability and Mathematical Statistics, John Wiley \& Sons Inc., New York, 1986. Characterization and convergence. MR838085 (88a:60130)

[ 3 ] K. Funano, Asymptotic behavior of mm-spaces, Doctoral Thesis, Tohoku University (2009).

[4] Andreas Greven, Pete Pfaffelhuber, and Anita Winter, Convergence in distribution of random metric measure spaces $(\Lambda$-coalescent measure trees $)$, Probab. Theory Related Fields 145 (2009), no. 1-2, 285-322, DOI 10.1007/s00440-008-0169-3. MR2520129 (2011c:60008)

[5] Misha Gromov, Metric structures for Riemannian and non-Riemannian spaces, Reprint of the 2001 English edition, Modern Birkhäuser Classics, Birkhäuser Boston, Inc., Boston, MA, 2007. Based on the 1981 French original; With appendices by M. Katz, P. Pansu and S. Semmes; Translated from the French by Sean Michael Bates. MR2307192 (2007k:53049) 
[6] Takefumi Kondo, Probability distribution of metric measure spaces, Differential Geom. Appl. 22 (2005), no. 2, 121-130, DOI 10.1016/j.difgeo.2004.10.001. MR2122737 (2006a:53039)

[ 7 ] Wolfgang Löhr, Equivalence of Gromov-Prohorov- and Gromov's $\square_{\lambda}$-metric on the space of metric measure spaces, Electron. Commun. Probab. 18 (2013), no. 17, 10. MR3037215

[ 8 ] A. M. Vershik, The universal Uryson space, Gromov's metric triples, and random metrics on the series of natural numbers, Uspekhi Mat. Nauk 53 (1998), no. 5(323), 57-64, DOI 10.1070/rm1998v053n05ABEH000069 (Russian); English transl., Russian Math. Surveys 53 (1998), no. 5, 921-928. MR1691182 (2000b:53055)

[9] Cédric Villani, Topics in optimal transportation, Graduate Studies in Mathematics, vol. 58, American Mathematical Society, Providence, RI, 2003. MR1964483 (2004e:90003)

Mathematical Institute

Tohoku University

Sendai 980-8578, Japan

E-mail:sb0m08@math.tohoku.ac.jp 